\title{
Contribution of Health Behavior to Emergence of Non-communicable Diseases: The Male Gender Norms Perspective
}

Faith Anuoluwapo Oluwadamilare ${ }^{1 *}$, Chiemeka Oscar Ezema ${ }^{2}$, Foluke Esther Akinleye ${ }^{3}$

${ }^{1}$ University of Ibadan, Ibadan, Oyo state, NIGERIA

2 Achieving Health Nigeria Initiative, Uyo, Akwa Ibom State, NIGERIA

3 Department of Clinical Nursing, University College Hospital, Ibadan, Oyo State, NIGERIA

* Corresponding author: damilare.faithy@gmail.com

\section{EUROPEAN JOURNAL OF BASIC MEDICAL SCIENCE}

Eur J Basic Med Sci 2018;8(1):1-4

Received: 12 Sep 2018

Accepted: 23 Nov 2018

Copyright ( $) 2018$ by Author/s and Licensed by Veritas Publications. This is an open access article distributed under the Creative Commons Attribution 4.0 International (CC BY 4.0) which permits unrestricted use, distribution, and reproduction in any medium, provided the original work is properly cited.

\begin{abstract}
Non-communicable diseases (NCDs) are a global health concern as they constitute a leading cause of death for both men and women globally. Noncommunicable diseases account for 41 million deaths per year and $71 \%$ of all deaths worldwide. In many societies, men tend to enjoy more privileges and opportunities than women, however, this does not necessarily result in better health outcomes for them. Masculinity in many cultures is characterized by riskenhancing behaviors such as intake of alcohol, smoking, self-medication and, lower rates of medical facility usage. These behaviors predispose to Noncommunicable diseases and directly influence the prevalence of the disease in the male gender. In this article, we explore the effect of gender characteristics on the prevalence of this increasing global health challenge.
\end{abstract}

Keywords: non-communicable diseases, risk factors for Non-communicable diseases, health-seeking behaviors in men 


\section{INTRODUCTION}

Non-communicable diseases (NCDs) are one of the major global health issues and have become a wellknown cause of mortality on a global scale. The top four main types of NCDs altogether account for more than $80 \%$ of all premature NCD deaths, with cardiovascular diseases accounting for the most deaths at (17.9 million deaths annually), followed by cancers ( 9.0 million), chronic respiratory diseases (3.9 million), and then diabetes (1.6 million) [1].

As a leading cause of death for both men and women, NCDs account for 41 million deaths per year and $71 \%$ of all deaths worldwide [1]. The burden of noncommunicable diseases is increasing globally and is known to affect men more than women with a ratio between 1 to 2 [2].

Short SE et al. defines health behaviors as "actions undertaken by individuals either intentional or unintentional to promote, maintain or enhance health and well-being and prevent disease or mortality" and are known to contribute to the development of NCDs in both men and women. They include "nutrition, exercise, sleep, sexual behavior, smoking, drug use, risk-taking adventures, medical service usage (physician usage, vaccination, screening) and compliance to medical treatments which vary among men and women" [3]. Public health experts tend to focus on these as individual behaviors that threaten health outcomes without necessarily linking them to gender norms.

Some of these health-related behaviors that affect the emergence of NCDs are further discussed below:

\section{Diet, Rest and, Physical Activity}

Diet plays a key role in health outcomes. The emergence of NCDs in boys and men can be considerably linked to dietary behaviors. Evidence gathered in the WHO men's health report shows that men are less responsive than women to foods promoted or labeled as healthier options and are less familiar with labeling schemes. They tend to consume more processed foods high in saturated fats, refined carbohydrates, low fiber, and other foods labeled as unhealthy [4].

Physical activity and diet are also strongly influenced by societal expectations of masculinities and femininities. Studies have shown that boys are more physically active than girls and that parents allowed independent mobility for boys than girls, putting girls more at risk for obesity than boys [5].

Hypercholesteremia and obesity are significant risk factors for hypertension and diabetes which result from physical inactivity and unhealthy eating habits. These may further lead to premature death from cardiovascular complications arising from these risk factors [6].

\section{Alcohol Consumption, Smoking and, Drug Use}

Tobacco use is associated with masculinity and consumption is higher in men than women. In many cultures, it is acceptable for men to consume large quantities of alcohol. A study carried out in the Russian Federation showed that heavy drinking of strong spirits "elevates or maintains a man's status in social groups" [7]. Alcohol and tobacco use put men at a heightened risk for developing certain types of NCDs such as hypertension, stroke, Chronic Obstructive Pulmonary Disease (COPD) and, cancer [8]. In 2010, 3.14 million men - as opposed to 1.72 million women - died from causes linked to excessive alcohol use. Also, 60 of 67 risk factors and risk factor clusters identified in the GBD 2010 study, were responsible for more male than female deaths and found to be more common in men were the top 10 risk factors [9].

\section{Sexual Behaviors}

Traditionally, males are expected to know more and are more experienced about sex, placing them at risk of contracting sexually transmitted infections because such norms keep them from seeking information and may lead them to engage in unsafe sexual practices [10]. In comparison with women, men tend to engage in early sexual intercourse, are more likely to have sex under the influence of drugs or alcohol, are less inclined to use a condom during sex, and have multiple sexual partners [11].

\section{Medical Service Usage}

Gender norms and roles also affect men's healthseeking behaviors and the responses they receive from services. Sex-aggregated data on patterns of healthseeking have shown that women utilize health care facilities more than men- mostly for women's use of sexual and reproductive health services [12]. 
Oluwadamilare et al.

Compared to men, women have a more positive attitude towards health and this may be influenced by several factors such as cultural norms and education.

Many studies emphasize that gender differences in health-seeking are influenced by, but not limited to cultural gender roles, economic factors and, gender power relations, but also unique to each population setting [13]. Men are also less likely to visit the hospital to report an illness and often opt for self-medication which may result in late detection and diagnosis of several diseases including Non-communicable diseases. This contributes to increased complications and mortality due to NCDs [14,15].

\section{Socio-Economic Determinants}

Socio-economic factors also play a role in determining how these gender norms affect health, as beliefs and expectations about men's behavior - for example, that men should be the sole provider of family income - can become a risk factor when exacerbated by lack of economic opportunities and social marginalization, thereby contributing to men's poor health behaviors and high rates of morbidity from preventable diseases, particularly suicide [16].

Furthermore, as a result of the highly gendered nature of employment, men tend to be more exposed to the development of occupationally related illnesses than females [17]. Working in high-risk environments such as mines and factories exponentially exposes men to the risk of the development of several NCDs including cancers and chronic respiratory diseases.

\section{CONCLUSION}

Non-communicable diseases have long been identified as a global health challenge and are a leading cause of mortality worldwide. To achieve the 2030 agenda for sustained development, this disease group must be overcome. However, this goal cannot be achieved if the influence of gender on risk factors for NCDs is overlooked. Men are more predisposed to NCDs, complications from NCDs and, death than women because risk-enhancing behaviors are more pronounced in this gender. It is therefore imperative to integrate gender analysis in health policies, research, and biomedical technologies to further improve the lives of men, and women globally.

Author contributions: All authors contributed equally. All authors confirmed the final article.
Conflict of Interest: None to Declare

Source of funding: No funding

\section{REFERENCES}

1. Forouzanfar M, Afshin A, Alexander LT, Anderson H, Bhutta Z, Murray CJL. GBD 2015 Risk Factors Collaborators. Global, regional, and national comparative risk assessment of 792 behavioural, environmental and occupational, and metabolic risks or clusters of risks, 1990-2015: a systematic analysis for the Global Burden of Disease Study 2015. Lancet. 2016; 388: 1659-724. doi: 10.1016/S0140-6736(16)31679-8.

2. Stevens A, Schmidt MI, Duncan BB. Gender inequalities in non-communicable disease mortality in Brazil. Cien Saude Colet. 2012 Oct; 17(10): 2627-34. doi: 10.1590/s141381232012001000012 PMID: 23099750.

3. Short SE, Mollborn S. Social Determinants and Health Behaviors: Conceptual Frames and Empirical Advances. Curr Opin Psychol. 2015; 5: 78-84. doi: 10.1016/j.copsyc.2015.05.002 PMid:26213711 PMCid:PMC4511598.

4. World Health Organization. The health and wellbeing of men in the WHO European Region: better health through a gender approach. 2018. Available at: https://www.euro.who.int/en/publications/ abstracts/the-health-and-well-being-of-men-inthe-who-european-region-better-health-througha-gender-approach-2018

5. Coen SE, Subedi RP, Rosenberg MW. Working out across Canada: Is there a gender gap? The Canadian Geographer / Le Géographe canadien, 2016; 60: 69-81. doi: 10.1111/cag. 12255.

6. WHO. Non-communicable diseases, 2018. Available at: https://www.who.int/news-room/factsheets/detail/noncommunicable-diseases

7. Hinote BP, Webber GR. Drinking toward manhood: masculinity and alcohol in the former USSR. Men Masc. 2012; 15: 292-310. doi: $10.1177 / 1097184 X 12448466$. 
8. Blomstrand A, Blomstrand C, Ariai N, Bengtsson C, Björkelund $C$. Stroke incidence and association with risk factors in women: A 32-year follow-up of the Prospective Population Study of Women in Gothenburg. BMJ Open. 2014; 4: e005173. doi: 10.1136/bmjopen-2014-005173 PMid:25351597 PMCid:PMC4212180.

9. Wang $\mathrm{H}$, Dwyer-Lindgren $\mathrm{L}$, Lofgren $\mathrm{KT}$, Rajaratnam JK, Marcus JR, Levin-Rector A, et al. Age-specific and sex-specific mortality in 187 countries, 1970-2010: a systematic analysis for the Global Burden of Disease Study 2010. Lancet. 2012; 380: 2071-94. doi: 10.1016/S0140-6736(12)61719$\mathrm{X}$.

10. Mane P, Gupta GR, Weiss E. Effective communication between partners: AIDS and risk reduction for women. AIDS. 1994; 8(Suppl. 1): S325-S331.

11. Courtenay WH. Behavioral factors associated with disease, injury, and death among men: Evidence and implications for prevention. Journal of Men's Studies. 2000; 9: 81-142. doi: 10.3149/jms.0901.81.

12. Nabalamba A, Millar WJ. Going to the doctor. Health Rep. 2007; 18(1): 23-35.
13. Das M, Angeli F, Krumeich AJSM, van Schayck OCP. The gendered experience with respect to healthseeking behaviour in an urban slum of Kolkata, India. International Journal for Equity in Health. 2018; 17: 24. doi: 10.1186/s12939-018-0738-8 PMid:29444674 PMCid:PMC5813424.

14. Song $Y$, Bian $Y$. Gender differences in the use of health care in China: cross-sectional analysis. Int $\mathrm{J}$ Equity Health. 2014 Jan 30; 13: 8. doi: 10.1186/1475-9276-13-8 PMid:24476343 PMCid: PMC3917614.

15. Gender, women and health. Available at: http://www.who.int/gender/genderandhealth/en

16. Etienne CF. Addressing masculinity and men's health to advance universal health and gender equality Rev Panam Salud Publica. 2018; 42: e196. doi: 10.26633/RPSR.2018.196.

17. Baker $\mathrm{P}$, Dworkin SL, Tong $\mathrm{S}$, Banks I, Shand $\mathrm{T}$, Yamey G. The men's health gap: men must be included in the global health equity agenda. Bulletin of the World Health Organization 2014; 92: 618-20. doi: 10.2471/BLT.13.132795 PMid:25197149 PMCid:PMC4147416. 